Article

\title{
Palladium-Catalyzed Heck Coupling Reaction of Aryl Bromides in Aqueous Media Using Tetrahydropyrimidinium Salts as Carbene Ligands
}

\author{
Sedat Yaşar ${ }^{1}$, Emine Özge Özcan ${ }^{2}$, Nevin Gürbüz ${ }^{2}$, Bekir Çetinkaya ${ }^{3}$ and İsmail Özdemir ${ }^{2, *}$ \\ 1 Department of Chemistry, Faculty of Science and Art, Gaziosmanpaşa University, 60240 Tokat, \\ Turkey; E-Mail: syasar44@gmail.com (S.Y.) \\ 2 Department of Chemistry, Faculty of Science and Art, İnönü University, 44280 Malatya, Turkey; \\ E-Mails: eozgeozcan@gmail.com (E.Ö.Ö.); ngurbuz@inonu.edu.tr (N.G.) \\ 3 Department of Chemistry, Faculty of Science, Ege University, 35100 İzmir, Turkey; \\ E-Mail: bekircetinkaya@ege.edu.tr (B.C.)
}

* Author to whom correspondence should be addressed; E-Mail: iozdemir@inonu.edu.tr; Tel.: +904223410212; Fax: +904223410212.

Received: 16 December 2009; in revised form: 1 January 2010 / Accepted: 12 January 2010 / Published: 28 January 2010

\begin{abstract}
An efficient and stereoselective catalytic system for the Heck cross coupling reaction using novel 1,3-dialkyl-3,4,5,6-tetrahydropyrimidinium salts (1, LHX) and $\mathrm{Pd}(\mathrm{OAc})_{2}$ loading has been reported. The palladium complexes derived from the salts $\mathbf{1 a - f}$ prepared in situ exhibit good catalytic activity in the Heck coupling reaction of aryl bromides under mild conditions.
\end{abstract}

Keywords: carbene; C-C coupling; palladium/tetrahydropyrimidinium; Heck coupling

\section{Introduction}

The Heck reaction, one of the most utilized cross-coupling reactions, is the palladium-catalyzed arylation of an olefin with an aryl halide under basic conditions. Since its independent discovery in the early 1970s by Heck [1] and Mizoroki [2], the Heck reaction has been widely used as a tool for organic synthesis because of its importance in the direct attachment of olefinic groups to aromatic 
rings [3-5]. Numerous review articles on various aspects of the Heck and other cross-coupling reactions with palladium catalysts have been published [6-9]. Many types of ligands have been explored for the palladium catalysts in the Heck reaction, e.g., phosphine [10-12], carbene [13], amine [14] and thiolate [15]. The use of immobilized [16] and non-immobilized [17] ligand free palladium salts for the Mizoroki-Heck reaction with chloro- and bromoarenes is also discussed in the literature. Recently, Gibson et al. [18] as well as Jones et al. [20] showed that palladium complexes with chiral ligands are well suited as catalysts for the asymmetric Heck reaction.

Heck coupling reactions often require high temperatures (normally $110-180{ }^{\circ} \mathrm{C}$ ) to proceed, even with activated aryl bromides [20-24]. Only a few catalyst systems can catalyze Heck-Mizoroki reactions at a temperature below $100{ }^{\circ} \mathrm{C}[25,26]$. The high thermal stability of transition metal complexes of $N$-heterocyclic carbenes (NHCs) makes these complexes particularly suitable for Heck coupling reactions [27-32]. The first application of $\mathrm{Pd}-\mathrm{NHC}$ complexes for the Heck reaction of aryl bromides and activated aryl chlorides was reported by Herrmann et al. in 1995 [13]. Since then, a number of monodentate and bidentate NHC ligands have been shown to have good activity in Pdcatalyzed Heck reactions [33-36]. Due to the high stability of metal complexes of NHCs towards heat, oxygen and moisture [37], they have long been the subject of catalytic studies. In addition to the palladium catalyzed Mizoroki-Heck reaction, they have been used for the catalysis of other $\mathrm{C}-\mathrm{C}$ coupling reactions [38], hydroformylations [39], polymerization reactions [40], olefin metathesis [41], and $\mathrm{CH}$-activation [42].

The nature of the NHC ligand has a tremendous influence on the rate of catalyzed reactions. Whilst modifications to the five membered ring of the ligand aryl substituent have been described, relatively little attention has been paid to effect of the ring size. Due to their six-membered ring geometry, tetrahydropyrimidine-2-ylidenes are stronger donating ligands in comparison to their five-membered relatives [43]. The use of tetrahydropyrimidinium ligands in catalytic transformations is limited [44-48].

We have previously reported the use of an in situ formed imidazolidine, tetrahydropyrimidine, tetrahydrodiazepine, benzimidazole-2-ylidene-palladium(II) system which exhibits high activity in various coupling reactions of aryl bromides and aryl chlorides [49-52]. In order to find more efficient palladium catalysts we have prepared a series of new 1,3-dialkyl-3,4,5,6-tetrahydropyrimidinium 1a-f (Scheme 1), and we now report the use of the in situ generated catalytic system composed of $\mathrm{Pd}(\mathrm{OAc})_{2}$ as palladium source, 1a-f as carbene precursors and $\mathrm{K}_{2} \mathrm{CO}_{3}$ as a base for cross coupling of aryl bromides with phenylstyrene in $\mathrm{DMF} /$ water at $80^{\circ} \mathrm{C}$ for $4 \mathrm{~h}$.

\section{Results and Discussion}

\subsection{Synthesis of tetrahydropyrimidinium salts}

A series of symmetrical 1,3-dialkyl-3,4,5,6-tetrahydropyrimidinium salts $(\mathbf{1}=\mathbf{L H X})$ were prepared according to known methods [28] as conventional NHC precursors. The symmetrical NHC precursors were prepared according to general reaction pathway depicted in Scheme 1. Treatment of 1,3-propylenediamine with 2 equivalents of aromatic aldehyde in methanol at room temperature led to the formation of the corresponding diimines. Their reduction with sodium borohydride in methanol, 
followed by treatment with triethylorthoformate in the presence of ammonium chloride with continuous elimination of ethanol led to the formation of the expected tetrahydropyrimidinium chlorides in excellent yields.

Scheme 1. Preparation of tetrahydropyrimidinium salts.

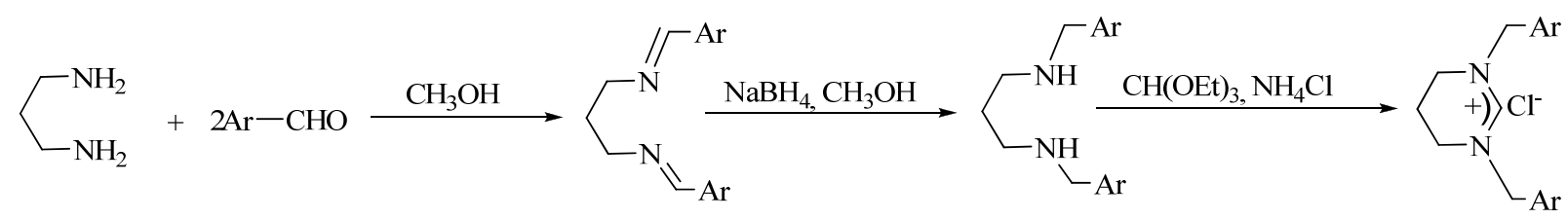

The tetrahydropyrimidinium salts (Figure 1) were isolated as colourless solids in very good yields and fully characterized by ${ }^{1} \mathrm{H}$ - and ${ }^{13} \mathrm{C}-\mathrm{NMR}$, and IR spectroscopies, elemental analyses, and their melting points were determined (see Experimental section).

Figure 1. Tetrahydropyrimidinium salts.

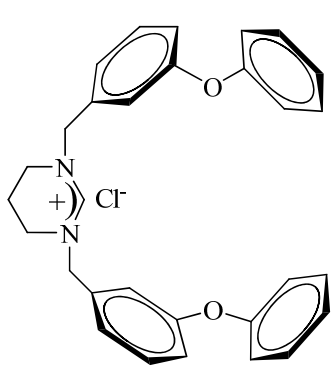

$1 \mathbf{a}$

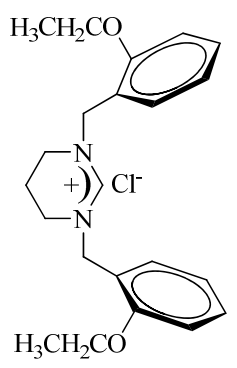

1d

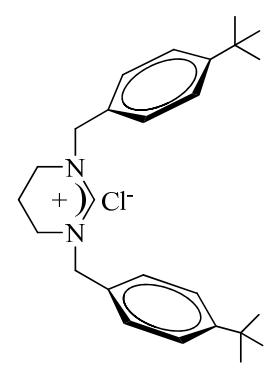

$1 \mathbf{b}$

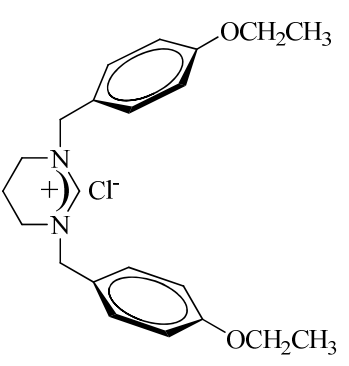

1e

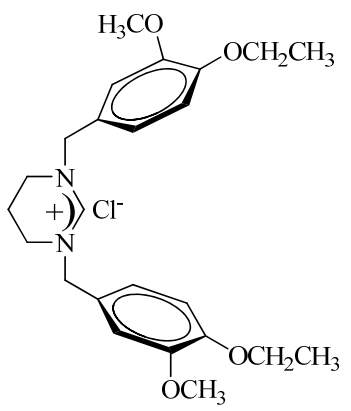

$1 \mathbf{c}$

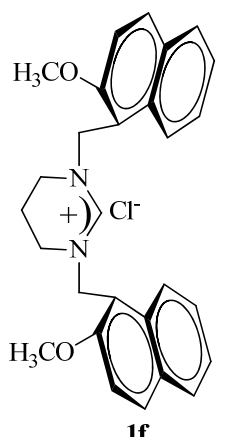

1f

The salts are air- and moisture stable both in the solid state and in solution. The NMR values are similar to those found for other tetrahydropyrimidinium salts [45]. Thus, the ${ }^{1} \mathrm{H}-\mathrm{NMR}$ spectra support the assigned structures; the resonances for the acidic C(2)-H in 1a-f were observed as sharp singlets at $10.28,10.20,10.08,9.40,10.45$ and $8.80 \mathrm{ppm}$, respectively. The position of the ethoxy groups on the phenyl rings also has a strong influence on the acidity of the proton as a chemical shift of $9.40 \mathrm{ppm}$ is observed in the case of the 2-substitution in $\mathbf{1 d}$, whereas the 4-substitution pattern in 1e leads to a signal at $10.45 \mathrm{ppm} .{ }^{13} \mathrm{C}-\mathrm{NMR}$ chemical shifts were also consistent with the proposed structures; the imino carbons of the tetrahydropyrimidinium salts 1a-f appeared as a typical singlet in the ${ }^{1} \mathrm{H}$ decoupled mode at 158.2, 154.5, 154.1, 157.4, 159.4 and 155.8 ppm, respectively. The IR data for 1a-f 
clearly indicate the presence of the $-\mathrm{C}=\mathrm{N}$ - group with $v(\mathrm{C}=\mathrm{N})$ vibrations at $1,695,1,701,1,695,1,688$ and $1,678 \mathrm{~cm}^{-1}$, respectively.

\subsection{The Heck C-C coupling reaction}

The Heck reaction has been shown to be very useful for the preparation of disubstituted olefins in particular. The rate of the coupling is dependent on a variety of parameters such as temperature, solvent, base and catalyst loading. Generally, Heck reactions conducted with tertiary phosphine or NHC complexes required high temperatures (higher than $120^{\circ} \mathrm{C}$ ) and polar solvents [20-24]. For the choice of base, we surveyed $\mathrm{Cs}_{2} \mathrm{CO}_{3}, \mathrm{~K}_{2} \mathrm{CO}_{3}, t-\mathrm{BuOK}$, and $\mathrm{K}_{3} \mathrm{PO}_{4}$. To find optimum conditions a series of experiments was performed with 4-bromoacetophenone and styrene as model compounds. Finally, we found that use of $1 \% \operatorname{mol} \mathrm{Pd}(\mathrm{OAc})_{2}, 2 \%$ mol 1,2 equiv $\mathrm{K}_{2} \mathrm{CO}_{3}$ in $\mathrm{DMF} / \mathrm{H}_{2} \mathrm{O}(1: 1)$ at $80{ }^{\circ} \mathrm{C}$ led to the best conversion within $4 \mathrm{~h}$. Since the salts 1a-f are thermally stable and inert toward air and moisture in the solid state, these properties allowed for catalytic experiments under aerobic conditions and the reactions were performed in air and without degassing the water and DMF prior to use.

Control experiment indicated that the coupling reaction did not occur in the absence of the salt $\mathbf{1}$. Under the determined reaction conditions, a wide range of aryl bromides bearing electron-donating or electron-withdrawing groups react with styrene affording the coupled products in optimum yields. As expected, the use of electron-deficient bromides was beneficial for the conversions. Under those conditions $p$-bromoacetophenone, $p$-bromobenzaldehyde, bromotoluene and $p$-bromoanisole react very cleanly and in goods yields with styrene (Table 1, entries 4, 10, 15 and 22). Enhancement in activity, although less significant, is further observed employing 4-bromo-benzaldehyde instead of 4bromoacetophenone (Table 1, entries 1-6, 19-24). The substituent effect in the tetrahydropyrimidinium salts indicated that a ethoxy group of the aromatic ring on the $N$ atoms increased the yield of product. The catalytic activity of the salts used falls in the order $\mathbf{b} \approx \mathbf{d}>\mathbf{c}>\mathbf{a}>$ e $>$ f. Table 1 summarizes our results from the screening of new tetrahydropyrimidinium salts for the Heck coupling reaction. The procedure is simple and does not require an induction period. This catalytic system provides good conditions for the coupling of aryl bromides under air. It should be noted that in all cases only the trans products were selectively obtained as confirmed by ${ }^{1} \mathrm{H}-\mathrm{NMR}$.

Table 1. The Heck coupling reaction of aryl bromides with styrene.

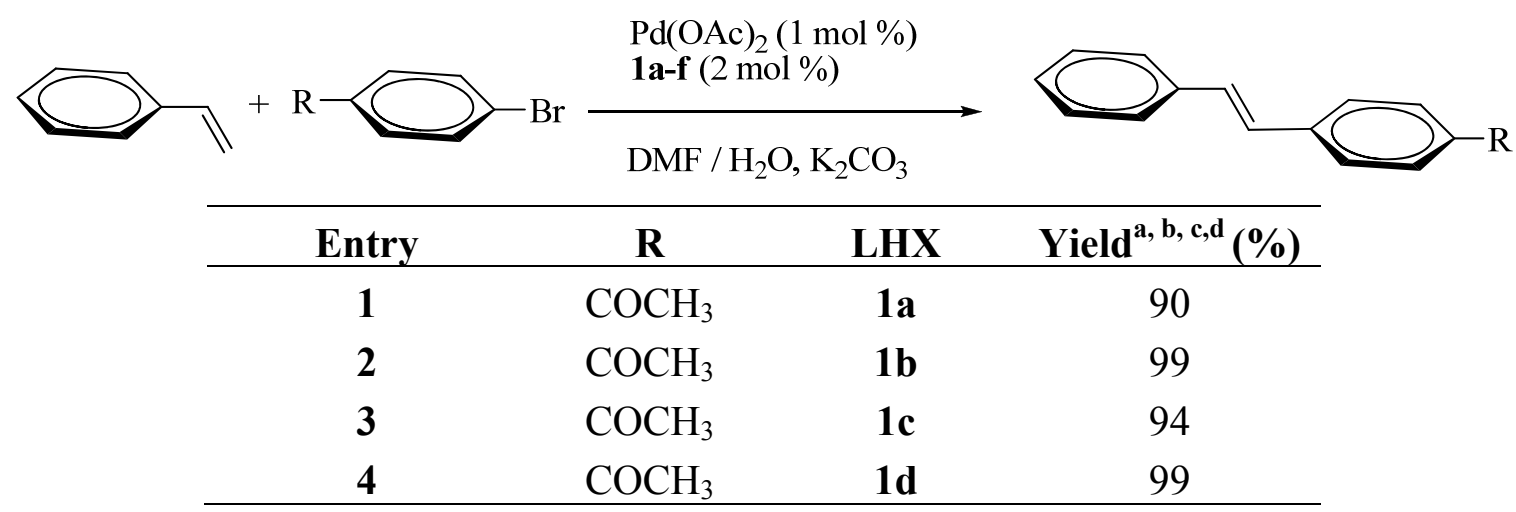


Table 1. Cont.

\begin{tabular}{|c|c|c|c|}
\hline 5 & $\mathrm{COCH}_{3}$ & $1 e$ & 93 \\
\hline 6 & $\mathrm{COCH}_{3}$ & 1f & 86 \\
\hline 7 & $\mathrm{CH}_{3}$ & $1 \mathbf{a}$ & 65 \\
\hline 8 & $\mathrm{CH}_{3}$ & $1 b$ & 82 \\
\hline 9 & $\mathrm{CH}_{3}$ & $1 c$ & 78 \\
\hline 10 & $\mathrm{CH}_{3}$ & $1 d$ & 95 \\
\hline 11 & $\mathrm{CH}_{3}$ & $1 e$ & 70 \\
\hline 12 & $\mathrm{CH}_{3}$ & 1f & 82 \\
\hline 13 & $\mathrm{OCH}_{3}$ & $1 \mathrm{a}$ & 70 \\
\hline 14 & $\mathrm{OCH}_{3}$ & $1 b$ & 96 \\
\hline 15 & $\mathrm{OCH}_{3}$ & $1 \mathrm{c}$ & 98 \\
\hline 16 & $\mathrm{OCH}_{3}$ & 1d & 99 \\
\hline 17 & $\mathrm{OCH}_{3}$ & 1e & 98 \\
\hline 18 & $\mathrm{OCH}_{3}$ & 1f & 74 \\
\hline 19 & $\mathrm{CHO}$ & $1 \mathrm{a}$ & 97 \\
\hline 20 & $\mathrm{CHO}$ & $1 b$ & 98 \\
\hline 21 & $\mathrm{CHO}$ & $1 \mathrm{c}$ & 97 \\
\hline 22 & $\mathrm{CHO}$ & 1d & 99 \\
\hline 23 & $\mathrm{CHO}$ & 1e & 96 \\
\hline 24 & $\mathrm{CHO}$ & 1f & 86 \\
\hline 25 & $\mathrm{H}$ & $1 \mathrm{a}$ & 62 \\
\hline 26 & $\mathrm{H}$ & $1 b$ & 60 \\
\hline 27 & $\mathrm{H}$ & $1 c$ & 66 \\
\hline 28 & $\mathrm{H}$ & 1d & 85 \\
\hline 29 & $\mathrm{H}$ & 1e & 78 \\
\hline 30 & $\mathrm{H}$ & 1f & 82 \\
\hline
\end{tabular}

${ }^{a}$ Reaction conditions: $1.0 \mathrm{mmol}$ of $\mathrm{R}-\mathrm{C}_{6} \mathrm{H}_{4} \mathrm{Br}-\mathrm{p}, 1.5 \mathrm{mmol}$ of styrene, $2 \mathrm{mmol} \mathrm{K} \mathrm{CO}_{3}, 1 \mathrm{mmol} \%$ $\mathrm{Pd}(\mathrm{OAc})_{2}, 2 \mathrm{mmol} \% \mathbf{1 a - f}, \mathrm{H}_{2} \mathrm{O}(3 \mathrm{~mL})$-DMF $(3 \mathrm{~mL})$; ${ }^{\mathrm{b}}$ Purity of compounds is checked by NMR and yields are based on aryl halide; ${ }^{\mathrm{c}}$ All reactions were monitored by TLC and GC; ${ }^{\mathrm{d}}$ Temperature $80^{\circ} \mathrm{C}, 4 \mathrm{~h}$.

\section{Experimental Section}

\subsection{General}

All reactions for the preparation of $\mathbf{1}$ were carried out under Ar in flame-dried glassware using standard Schlenk-type flasks. The solvents used were purified by distillation over the indicated drying agents and were transferred under $\mathrm{Ar}$ : $\mathrm{Et}_{2} \mathrm{O}\left(\mathrm{Na} / \mathrm{K}\right.$ alloy), $\mathrm{C}_{2} \mathrm{H}_{5} \mathrm{OH}(\mathrm{Mg})$. Flash chromatography: Merck silica gel 60 (230-400 mesh). All reagents were purchased from Aldrich Chemical Co. Melting point were determined in glass capillaries under air with an Electrothermal-9200 melting point 
apparatus. FT-IR spectra were recorded as $\mathrm{KBr}$ pellets in the range $400-4,000 \mathrm{~cm}^{-1}$ on a ATI UNICAM 1000 spectrometer. ${ }^{1} \mathrm{H}-\mathrm{NMR}$ and ${ }^{13} \mathrm{C}$-NMR spectra were recorded in $\mathrm{CDCl}_{3}$ with tetramethylsilane as an internal reference using a Varian As 400 Merkur spectrometer operating at $400 \mathrm{MHz}\left({ }^{1} \mathrm{H}\right)$, $100 \mathrm{MHz}\left({ }^{13} \mathrm{C}\right)$. The NMR studies were carried out in high-quality $5 \mathrm{~mm}$ NMR tubes. Signals are quoted in parts per million as $\delta$ downfield from tetramethylsilane $(\delta 0.00)$ as an internal standard. Coupling constants ( $J$ values) are given in hertz. NMR multiplicities are abbreviated as follows: $\mathrm{s}=$ singlet, $\mathrm{d}=$ doublet, $\mathrm{t}=$ triplet, $\mathrm{m}=$ multiplet signal. All reactions were monitored on an Agilent $6890 \mathrm{~N}$ GC system by GC-FID with a HP-5 column of $30 \mathrm{~m}$ length, $0.32 \mathrm{~mm}$ diameter and $0.25 \mu \mathrm{m}$ film thickness. Column chromatography was performed using silica gel 60 (70-230 mesh). Solvent ratios are given as v/v. Elemental analyses were performed by Turkish Research Council Microlab (Ankara, Turkey).

\subsection{General preparation of symmetrical 1,3-dialkyl-3,4,5,6-tetrahydropyrimidinium salts}

The aromatic aldehyde $(20 \mathrm{mmol})$ and 1,3-propylendiamine $(10 \mathrm{mmol})$ were stirred overnight in methanol $(25 \mathrm{~mL})$. The diimine was collected as a white solid, filtered and recrystallized from an alcohol/ether mixture. The diimine $(10 \mathrm{mmol})$ was subsequently reduced by $\mathrm{NaBH}_{4}(30 \mathrm{mmol})$ in $\mathrm{CH}_{3} \mathrm{OH}(30 \mathrm{~mL})$. The solution was then treated with $1 \mathrm{~N} \mathrm{HCl}$, and the organic phase was extracted with $\mathrm{CH}_{2} \mathrm{Cl}_{2}(3 \times 30 \mathrm{~mL})$. After drying over $\mathrm{MgSO}_{4}$ and evaporation, the diamine was isolated as a solid and then treated in a large excess of triethyl orthoformate $(50 \mathrm{~mL})$ in the presence of $\mathrm{NH}_{4} \mathrm{Cl}$ $(10 \mathrm{mmol})$ at $110^{\circ} \mathrm{C}$ in a distillation apparatus until the removal of ethanol ceased. Upon cooling to RT a colourless solid precipitated, which was collected by filtration and dried under vacuum. The crude product was recrystallized from absolute ethanol to give colourless needles and the solid was washed with diethyl ether $(2 \times 10 \mathrm{~mL})$ and dried under vacuum.

1,3-Bis-(3-phenoxybenzyl)-3,4,5,6-tetrahydropyrimidinium chloride (1a). Yield: 87\%, m.p.: 125-126 ${ }^{\circ} \mathrm{C} ; \mathrm{v}_{(\mathrm{CN})}=1,695 \mathrm{~cm}^{-1} ;{ }^{1} \mathrm{H}-\mathrm{NMR} \delta: 1.19$ (quint, $J=4 \mathrm{~Hz}, 2 \mathrm{H}, \mathrm{NCH}_{2} \mathrm{CH}_{2} \mathrm{CH}_{2} \mathrm{~N}$ ), 3.23 (t, $\left.J=4 \mathrm{~Hz}, 4 \mathrm{H}, \mathrm{NCH}_{2} \mathrm{CH}_{2} \mathrm{CH}_{2} \mathrm{~N}\right), 4.89\left(\mathrm{~s}, 4 \mathrm{H}, \mathrm{CH}_{2} \mathrm{C}_{6} \mathrm{H}_{4} \mathrm{OC}_{6} \mathrm{H}_{5}\right), 6.89-7.33\left(\mathrm{~m}, 18 \mathrm{H}, \mathrm{CH}_{2} \mathrm{C}_{6} \mathrm{H}_{4} \mathrm{OC}_{6} \mathrm{H}_{5}\right)$, $10.28(\mathrm{~s}, 1 \mathrm{H}, \quad \mathrm{NCHN}) ;{ }^{13} \mathrm{C}\{\mathrm{H}\} \mathrm{NMR} \delta: 19.2\left(\mathrm{NCH}_{2} \mathrm{CH}_{2} \mathrm{CH}_{2} \mathrm{~N}\right), 42.2\left(\mathrm{NCH}_{2} \mathrm{CH}_{2} \mathrm{CH}_{2} \mathrm{~N}\right), 58.4$ $\left(\mathrm{CH}_{2} \mathrm{C}_{6} \mathrm{H}_{4} \mathrm{OC}_{6} \mathrm{H}_{5}\right), 118.9,119.4,123.4,124.0,130.1,131.0,135.4,155.2$ and $156.7\left(\mathrm{CH}_{2} \mathrm{C}_{6} \mathrm{H}_{4} \mathrm{OC}_{6} \mathrm{H}_{5}\right)$, $158.2(\mathrm{NCHN})$; Anal. Calcd. for $\mathrm{C}_{30} \mathrm{H}_{29} \mathrm{~N}_{2} \mathrm{O}_{2} \mathrm{Cl}$ : C,74.29; H, 6.03; N, 5.78\%; found: C, 74.32; H, 6.08; $\mathrm{N}, 5.83 \%$.

1,3-Bis-(4-t-butylbenzyl)-3,4,5,6-tetrahydropyrimidinium chloride (1b). Yield: 85\%, m.p.: $315{ }^{\circ} \mathrm{C}$; $\mathrm{v}_{(\mathrm{CN})}=1,701 \mathrm{~cm}^{-1} ;{ }^{1} \mathrm{H}-\mathrm{NMR} \delta: 1.22\left(\mathrm{~s}, 18 \mathrm{H}, \mathrm{CH}_{2} \mathrm{C}_{6} \mathrm{H}_{4} \mathrm{C}\left(\mathrm{CH}_{3}\right)_{3}-4\right), 1.90$ (quint, $\mathrm{J}=4 \mathrm{~Hz}, 2 \mathrm{H}$, $\mathrm{NCH}_{2} \mathrm{CH}_{2} \mathrm{CH}_{2} \mathrm{~N}$ ), 3.16 (t, $\left.J=4 \mathrm{~Hz}, 4 \mathrm{H}, \mathrm{NCH}_{2} \mathrm{CH}_{2} \mathrm{CH}_{2} \mathrm{~N}\right), 4.81$ (s, 4H, $\left.\mathrm{CH}_{2} \mathrm{C}_{6} \mathrm{H}_{4} \mathrm{C}\left(\mathrm{CH}_{3}\right)_{3}-4\right), 7.28$ (m, $\left.8 \mathrm{H}, \mathrm{CH}_{2} \mathrm{C}_{6} \mathrm{H}_{4} \mathrm{C}\left(\mathrm{CH}_{3}\right)_{3}-4\right), 10.20(\mathrm{~s}, 1 \mathrm{H}, \mathrm{NCHN}) ;{ }^{13} \mathrm{C}\{\mathrm{H}\} \mathrm{NMR} \delta: 19.2\left(\mathrm{NCH}_{2} \mathrm{CH}_{2} \mathrm{CH}_{2} \mathrm{~N}\right), 31.5$

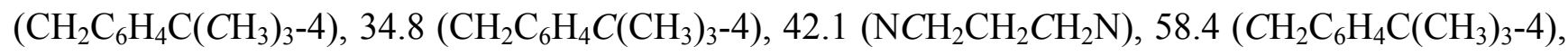
126.2, 128.7, 130.4 and 152.1 $\left(\mathrm{CH}_{2} \mathrm{C}_{6} \mathrm{H}_{4} \mathrm{C}\left(\mathrm{CH}_{3}\right)_{3}-4\right), 154.5(\mathrm{NCHN})$; Anal. Calcd. for $\mathrm{C}_{26} \mathrm{H}_{37} \mathrm{~N}_{2} \mathrm{Cl}$ : C,75.61; H, 9.03; N, 6.78\%; found: C, 75.66; H, 9.08; N, 6.83\%. 
1,3-Bis-(3-methoxy-4-ethoxybenzyl)-3,4,5,6-tetrahydropyrimidinium chloride (1c). Yield: 89\%, m.p.: 177-178 ${ }^{\circ} \mathrm{C} ; \mathrm{U}_{(\mathrm{CN})}=1,695 \mathrm{~cm}^{-1} ;{ }^{1} \mathrm{H}-\mathrm{NMR} \delta: 1.35\left(\mathrm{t}, J=4.8 \mathrm{~Hz}, 6 \mathrm{H}_{,} \mathrm{CH}_{2} \mathrm{C}_{6} \mathrm{H}_{3} \mathrm{OCH}_{2} \mathrm{CH}_{3}-4\right), 1.85$ (quint, $\left.J=5.6 \mathrm{~Hz}, 2 \mathrm{H}, \mathrm{NCH}_{2} \mathrm{CH}_{2} \mathrm{CH}_{2} \mathrm{~N}\right), 3.14\left(\mathrm{t}, J=5.6 \mathrm{~Hz}, 4 \mathrm{H}, \mathrm{NCH}_{2} \mathrm{CH}_{2} \mathrm{CH}_{2} \mathrm{~N}\right), 3.81(\mathrm{~s}, 6 \mathrm{H}$, $\mathrm{CH}_{2} \mathrm{C}_{6} \mathrm{H}_{3} \mathrm{OCH}_{3}-3$ ), 3.98 (quart., $\left.J=4.8 \mathrm{~Hz}, 4 \mathrm{H}, \mathrm{CH}_{2} \mathrm{C}_{6} \mathrm{H}_{3} \mathrm{OCH}_{2} \mathrm{CH}_{3}-4\right), 4.71$ (s, $4 \mathrm{H}, \mathrm{CH}_{2} \mathrm{C}_{6} \mathrm{H}_{3}\left(\mathrm{OCH}_{3}-\right.$ 3)- $\left.\left(\mathrm{OCH}_{2} \mathrm{CH}_{3}-4\right)\right)$, 6.70-7.05 (m, 6H, $\left.\mathrm{CH}_{2} \mathrm{C}_{6} \mathrm{H}_{3}\left(\mathrm{OCH}_{3}-3\right)-\left(\mathrm{OCH}_{2} \mathrm{CH}_{3}-4\right)\right), 10.08$ (s, $\left.1 \mathrm{H}, \mathrm{NCHN}\right)$; ${ }^{13} \mathrm{C}\{\mathrm{H}\} \mathrm{NMR} \delta: 14.9\left(\mathrm{CH}_{2} \mathrm{C}_{6} \mathrm{H}_{3} \mathrm{OCH}_{2} \mathrm{CH}_{3}-4\right), 19.2\left(\mathrm{NCH}_{2} \mathrm{CH}_{2} \mathrm{CH}_{2} \mathrm{~N}\right), 41.9\left(\mathrm{NCH}_{2} \mathrm{CH}_{2} \mathrm{CH}_{2} \mathrm{~N}\right), 58.5$ $\left(\mathrm{CH}_{2} \mathrm{C}_{6} \mathrm{H}_{3}\left(\mathrm{OCH}_{3}-3\right)-\left(\mathrm{OCH}_{2} \mathrm{CH}_{3}-4\right)\right), 56.7\left(\mathrm{CH}_{2} \mathrm{C}_{6} \mathrm{H}_{3} \mathrm{OCH}_{2} \mathrm{CH}_{3}-4\right), 64.5\left(\mathrm{CH}_{2} \mathrm{C}_{6} \mathrm{H}_{3} \mathrm{OCH}_{3}-3\right)$, 121.6, 125.9, 149.0 and $150.0\left(\mathrm{CH}_{2} \mathrm{C}_{6} \mathrm{H}_{3}\left(\mathrm{OCH}_{3}-3\right)-\left(\mathrm{OCH}_{2} \mathrm{CH}_{3}-4\right)\right)$, 154.1 (NCHN); Anal. Calcd. for $\mathrm{C}_{24} \mathrm{H}_{34} \mathrm{~N}_{2} \mathrm{O}_{4} \mathrm{Cl}$ : C,64.20; H, 7.41; N, 6.24\%; found: C, 64.24; H,7.44; N, 6.27\%.

1,3-Bis-(2-ethoxybenzyl)-3,4,5,6-tetrahydropyrimidinium chloride (1d). Yield: 80 \%, m.p.: 171-172 ${ }^{\circ} \mathrm{C} ; \mathrm{v}_{(\mathrm{CN})}=1,677 \mathrm{~cm}^{-1} ;{ }^{1} \mathrm{H}-\mathrm{NMR} \delta: 1.32\left(\mathrm{t}, \mathrm{J}=8 \mathrm{~Hz}, 6 \mathrm{H}_{,} \mathrm{CH}_{2} \mathrm{C}_{6} \mathrm{H}_{4} \mathrm{OCH}_{2} \mathrm{CH}_{3}-2\right.$ ), 1.94 (quint, $J=4 \mathrm{~Hz}, 2 \mathrm{H}, \mathrm{NCH}_{2} \mathrm{CH}_{2} \mathrm{CH}_{2} \mathrm{~N}$ ), $3.27\left(\mathrm{t}, J=4 \mathrm{~Hz}, 4 \mathrm{H}, \mathrm{NCH}_{2} \mathrm{CH}_{2} \mathrm{CH}_{2} \mathrm{~N}\right.$ ), 3.98 (quart., $J=8 \mathrm{~Hz}, 4 \mathrm{H}$, $\mathrm{CH}_{2} \mathrm{C}_{6} \mathrm{H}_{4} \mathrm{OCH}_{2} \mathrm{CH}_{3}-2$ ), 4.79 (s, $\left.4 \mathrm{H}, \mathrm{CH}_{2} \mathrm{C}_{6} \mathrm{H}_{4} \mathrm{OCH}_{2} \mathrm{CH}_{3}-2\right)$, 6.81-7.42 (m, 8H, $\mathrm{CH}_{2} \mathrm{C}_{6} \mathrm{H}_{4} \mathrm{OCH}_{2} \mathrm{CH}_{3}-2$ ), $9.40(\mathrm{~s}, 1 \mathrm{H}, \mathrm{NCHN}) ;{ }^{13} \mathrm{C}\{\mathrm{H}\} \mathrm{NMR} \delta: 15.1\left(\mathrm{CH}_{2} \mathrm{C}_{6} \mathrm{H}_{4} \mathrm{OCH}_{2} \mathrm{CH}_{3}-2\right), 19.4\left(\mathrm{NCH}_{2} \mathrm{CH}_{2} \mathrm{CH}_{2} \mathrm{~N}\right), 42.7$ $\left(\mathrm{NCH}_{2} \mathrm{CH}_{2} \mathrm{CH}_{2} \mathrm{~N}\right), 54.3\left(\mathrm{CH}_{2} \mathrm{C}_{6} \mathrm{H}_{4} \mathrm{OCH}_{2} \mathrm{CH}_{3}-2\right), 64.0\left(\mathrm{CH}_{2} \mathrm{C}_{6} \mathrm{H}_{4} \mathrm{OCH}_{2} \mathrm{CH}_{3}-2\right), 121.2,121.8,130.8$, 131.6 and $154.7\left(\mathrm{CH}_{2} \mathrm{C}_{6} \mathrm{H}_{4} \mathrm{OCH}_{2} \mathrm{CH}_{3}-2\right), 157.4(\mathrm{NCHN})$; Anal. Calcd. for $\mathrm{C}_{22} \mathrm{H}_{29} \mathrm{~N}_{2} \mathrm{O}_{2} \mathrm{Cl}$ : C, 62.94; $\mathrm{H}$, 7.52; N, 7.20\%; found: C, 62.88; H, 7.54; N, 7.25\%.

1,3-Bis-(4-ethoxybenzyl)-3,4,5,6-tetrahydropyrimidinium chloride (1e). Yield: 85\%, m.p.: 199-200 ${ }^{\circ} \mathrm{C}$; $\mathrm{v}_{(\mathrm{CN})}=1,688 \mathrm{~cm}^{-1} ;{ }^{1} \mathrm{H}-\mathrm{NMR} \delta: 1.34\left(\mathrm{t}, J=6.9 \mathrm{~Hz}, 6 \mathrm{H}_{,} \mathrm{CH}_{2} \mathrm{C}_{6} \mathrm{H}_{4} \mathrm{OCH}_{2} \mathrm{CH}_{3}-4\right), 1.88$ (quint, $J=6 \mathrm{~Hz}$, $2 \mathrm{H}, \mathrm{NCH}_{2} \mathrm{CH}_{2} \mathrm{CH}_{2} \mathrm{~N}$ ), 3.14 (t, $J=5.7 \mathrm{~Hz}, 4 \mathrm{H}, \mathrm{NCH}_{2} \mathrm{CH}_{2} \mathrm{CH}_{2} \mathrm{~N}$ ), 3.36 (quart., $J=6.9 \mathrm{~Hz}, 4 \mathrm{H}$, $\left.\mathrm{CH}_{2} \mathrm{C}_{6} \mathrm{H}_{4} \mathrm{OCH}_{2} \mathrm{CH}_{3}-4\right), 4.75$ (s, 4H, $\left.\mathrm{CH}_{2} \mathrm{C}_{6} \mathrm{H}_{4} \mathrm{OCH}_{2} \mathrm{CH}_{3}-4\right), 6.77$ and 7.31 (d, J = $6 \mathrm{~Hz}, 8 \mathrm{H}$, $\left.\mathrm{CH}_{2} \mathrm{C}_{6} \mathrm{H}_{4} \mathrm{OCH}_{2} \mathrm{CH}_{3}-4\right), 10.45(\mathrm{~s}, 1 \mathrm{H}, \mathrm{NCHN}) ;{ }^{13} \mathrm{C}\{\mathrm{H}\} \mathrm{NMR} \delta: 14.7\left(\mathrm{CH}_{2} \mathrm{C}_{6} \mathrm{H}_{4} \mathrm{OCH}_{2} \mathrm{CH}_{3}-4\right), 18.9$ $\left(\mathrm{NCH}_{2} \mathrm{CH}_{2} \mathrm{CH}_{2} \mathrm{~N}\right), 41.6\left(\mathrm{NCH}_{2} \mathrm{CH}_{2} \mathrm{CH}_{2} \mathrm{~N}\right), 57.8\left(\mathrm{CH}_{2} \mathrm{C}_{6} \mathrm{H}_{4} \mathrm{OCH}_{2} \mathrm{CH}_{3}-4\right), 63.5\left(\mathrm{CH}_{2} \mathrm{C}_{6} \mathrm{H}_{4} \mathrm{OCH}_{2} \mathrm{CH}_{3}-4\right)$, 114.9, 124.9130 .3 and $153.8\left(\mathrm{CH}_{2} \mathrm{C}_{6} \mathrm{H}_{4} \mathrm{OCH}_{2} \mathrm{CH}_{3}-4\right)$, 159.4 (NCHN); Anal. Calcd. for $\mathrm{C}_{22} \mathrm{H}_{29} \mathrm{~N}_{2} \mathrm{O}_{2} \mathrm{Cl}$ : C,62.94; H, 7.52; N, 7.20\%; found: C, 62.89; H, 7.53; N, 7.23\%.

1,3-Bis-(2-methoxynaphtomethyl)-3,4,5,6-tetrahydropyrimidinium chloride (1f). Yield: 85\%, m.p.: $96-97{ }^{\circ} \mathrm{C} ; \mathrm{v}_{(\mathrm{CN})}=1,678 \mathrm{~cm}^{-1} .{ }^{1} \mathrm{H}-\mathrm{NMR} \delta: 1.88$ (quint, $J=6 \mathrm{~Hz}, 2 \mathrm{H}, \mathrm{NCH}_{2} \mathrm{CH}_{2} \mathrm{CH}_{2} \mathrm{~N}$ ), 3.24 (t, $J$ $\left.=5.7 \mathrm{~Hz}, 4 \mathrm{H}, \mathrm{NCH}_{2} \mathrm{CH}_{2} \mathrm{CH}_{2} \mathrm{~N}\right), 3.70\left(\mathrm{~s}, 6 \mathrm{H}, \mathrm{CH}_{2} \mathrm{C}_{10} \mathrm{H}_{6} \mathrm{OCH}_{3}-2\right), 5.08\left(\mathrm{~s}, 4 \mathrm{H}, \mathrm{CH}_{2} \mathrm{C}_{10} \mathrm{H}_{6} \mathrm{OCH}_{3}-2\right)$, 7.09-7.94 (m, $\left.12 \mathrm{H}, \mathrm{CH}_{2} \mathrm{C}_{10} \mathrm{H}_{6} \mathrm{OCH}_{3}-2\right), 8.80(\mathrm{~s}, 1 \mathrm{H}, \mathrm{NCHN}) ;{ }^{13} \mathrm{C}\{\mathrm{H}\} \mathrm{NMR} \delta: 18.0\left(\mathrm{NCH}_{2} \mathrm{CH}_{2} \mathrm{CH}_{2} \mathrm{~N}\right)$, $42.3\left(\mathrm{NCH}_{2} \mathrm{CH}_{2} \mathrm{CH}_{2} \mathrm{~N}\right), 48.8\left(\mathrm{CH}_{2} \mathrm{C}_{10} \mathrm{H}_{6} \mathrm{OCH}_{3}-2\right), 55.8\left(\mathrm{CH}_{2} \mathrm{C}_{10} \mathrm{H}_{6} \mathrm{OCH}_{3}-2\right), 111.4,112.0,121.5$, 123.6, 127.7, 128.4, 128.5, 131.5, 132.2 and $151.5\left(\mathrm{CH}_{2} \mathrm{C}_{10} \mathrm{H}_{6} \mathrm{OCH}_{3}-2\right), 155.8(\mathrm{NCHN})$; Anal. Calcd. for $\mathrm{C}_{28} \mathrm{H}_{29} \mathrm{~N}_{2} \mathrm{O}_{2} \mathrm{Cl}$ : C,72.95; H, 6.34; N, 6.08\%; found: C, 72.99; H, 6.38; N, 6.11\%.

\subsection{General procedure for the Heck Coupling reaction}

$\mathrm{Pd}(\mathrm{OAc})_{2}(1.0 \mathrm{mmol} \%)$, 1,3-dialkyl-3,4,5,6-tetrahydropyrimidinium salt 1 (2 mmol \%), aryl bromide $(1.0 \mathrm{mmol})$, styrene $(1.5 \mathrm{mmol}), \mathrm{K}_{2} \mathrm{CO}_{3}(2 \mathrm{mmol})$ water $(3 \mathrm{~mL})$-DMF $(3 \mathrm{~mL})$ were added to a small Schlenk tube and the mixture was heated at $80{ }^{\circ} \mathrm{C}$ for $4 \mathrm{~h}$. At the conclusion of the reaction, the mixture was cooled, extracted with ethyl acetate/hexane (1:5), filtered through a pad of silicagel with 
copious washing, concentrated and purified by flash chromatography on silica gel. Purity of the compounds was checked by NMR and GC; yields are based on aryl bromide.

\section{Conclusions}

In summary, we have synthesized new 3,4,5,6-tetrahydropyrimidinium salts as precursors of $N$ heterocyclic carbenes upon deprotonation. They have been associated with $\mathrm{Pd}(\mathrm{OAc})_{2}$ to generate catalytic species. Such $\mathrm{Pd}(\mathrm{OAc})_{2}$ - tetrahydropyrimidinium salts system could successfully catalyze the Heck reactions available for an array of substrates including a wide range of aryl bromides bearing electron-donating or electron-withdrawing groups with high activity and stability in aerial atmosphere. Detailed investigations focusing on NHC (imidazolidine, tetrahydropyrimidine, benzimidazolidine) and catalytic activity in this and other coupling reactions are ongoing.

\section{Acknowledgements}

We grateful to Technological and Scientific Research Council of Turkey TUBİTAK-CNRS (France) [TBAG-U/181 (106T716)] and Inönü University Research Fund (BAP 2009/13) for financial support.

\section{References and Notes}

1. Heck, R.F.; Nolley, J.P. Palladium-catalyzed vinylic hydrogen substitution reactions with aryl, benzyl, and styryl halides. J. Org. Chem. 1972, 37, 2320-2322.

2. Mizoroki, T.; Mori, K.; Ozaki, A. Arylation of olefin with aryl iodide catalyzed by palladium. Bull. Chem. Soc. Jpn. 1971, 44, 581-581.

3. Sakoda, K.; Mihara, J.; Ichikawa, J. Heck-type 5-endo-trig cyclization promoted by vinylic fluorines: Synthesis of 5-fluoro-3H-pyrroles. Chem. Commun. 2005, 4684-4686.

4. Dounay, A.B.; Overman, L.E.; Wrobleski, A.D. Sequential catalytic asymmetric Heck-iminium ion cyclization: Enantioselective total synthesis of the Strychnos alkaloid minfiensine. J. Am. Chem. Soc. 2005, 127, 10186-10187.

5. De Meijere, A.; Meyer, F.E. Fine feathers make fine birds: The Heck reaction in modern garb. Angew. Chem. Int. Ed. 1995, 33, 2379-2411.

6. Trzeciak, A.M.; Ziołkowski, J.J. Monomolecular, nanosized and heterogenized palladium catalysts for the Heck reaction. Coord. Chem. Rev. 2007, 251, 1281-1293.

7. Yin, L.; Liebscher, J. Carbon-carbon coupling reactions catalyzed by heterogeneous palladium catalysts. Chem. Rev. 2007, 107, 133-173.

8. Bedford, R.B.; Cazin, C.S.J.; Holder, D. Structural and mechanistic studies of Pd-catalyzed C-C bond formation: The case of carbonylation and Heck reaction. Coord. Chem. Rev. 2004, 248, 2283-2321.

9. Littke, A.F.; Fu, G.C. Palladium-catalyzed coupling reactions of aryl chlorides. Angew. Chem. Int. Ed. 2002, 41, 4126-4211. 
10. Littke, A.F.; Fu, G.C. Heck reactions in the presence of $\mathrm{P}(t-\mathrm{Bu})_{3}$ : Expanded scope and milder reaction conditions for the coupling of aryl chlorides. J. Org. Chem. 1999, 64, 10-11.

11. Shaughnessy, K.H.; Kim, P.; Hartwig, J.F. A Fluorescence-based assay for high-throughput screening of coupling reactions. Application to Heck chemistry. J. Am. Chem. Soc. 1999, 121, 2123-2132.

12. Ehrentraut, A.; Zapf, A.; Beller, M. A new efficient palladium catalyst for Heck reactions of deactivated aryl chlorides. Synlett 2000, 1589-1592.

13. Herrmann, W.A.; Elison, M.; Fischer, J.; Köcher, C.; Artus, G.R.J. Metal complexes of $N$ heterocyclic carbenes - A new structural principle for catalysts in homogeneous catalysis. Angew. Chem. Int. Ed. 1995, 34, 2371-2374.

14. Bedford, R.B. Palladacyclic catalysts in $\mathrm{C}-\mathrm{C}$ and $\mathrm{C}-$ heteroatom bond-forming reactions. Chem. Commun. 2003, 1787-1796.

15. Bergbreiter, D.E.; Osburn, P.L.; Liu, Y.S. Tridentate SCS palladium(II) complexes: New, highly stable, recyclable catalysts for the Heck reaction. J. Am. Chem. Soc. 1999, 121, 9531-9538.

16. Crudden, C.M.; Sateesh, M.; Lewis, R. Mercaptopropyl-modified mesoporous silica: A remarkable support for the preparation of a reusable, heterogeneous palladium catalyst for coupling reactions. J. Am. Chem. Soc. 2005, 127, 10045-10050.

17. De Vries, A.H.M.; Mulders, J.M.C.A.; Mommers, J.H.M.; Henderickx, H.J.W.; de Vries, J.G. Homeopathic ligand-free palladium as a catalyst in the Heck reaction. A comparison with a palladacycle. Org. Lett. 2003, 5, 3285-3288.

18. Gibson, S.E.; Ibrahim, H.; Pasquier, C.; Swamy, V.M. Novel planar chiral diphosphines and their application in asymmetric hydrogenations and asymmetric Heck reactions. TetrahedronAsymmetry 2004, 15, 465-473.

19. Dodd, D.W.; Toews, H.E.; Carneiro, F.D.S.; Jennings, M.C.; Jones, N.D. Model intermolecular asymmetric Heck reactions catalyzed by chiral pyridyloxazoline palladium(II) complexes. Inorg. Chim. Acta 2006, 359, 2850-2858.

20. Kantchev, E.A.B.; O’Brien, C.J.; Organ, M.G. Palladium complexes of $N$-heterocyclic carbenes as catalysts for cross-coupling reactions - A synthetic chemist's perspective. Angew. Chem. Int. Ed. 2007, 46, 2768-2813.

21. Brase, S.; de Meijere, A. In Metal-Catalyzed Cross-Coupling Reactions, Ed.; de Meijere A., Diederich F.; Wiley-VCH: Weinheim, Germany, 2004; pp. 217-315.

22. Iwasawa, T.; Komano, T.; Tajima, M.; Tokunaga, A.; Obora, Y.; Fujihara, T.; Tsuji, Y. Phosphines having a 2,3,4,5-tetraphenylphenyl moiety: Effective ligands in palladium-catalyzed transformations of aryl chlorides. Organometallics 2006, 25, 4665-4669.

23. Ebran, J.P.; Hansen, A.L.; Thomas M.; Gøgsig, T.M.; Skrydstrup, T. Studies on the Heck reaction with alkenyl phosphates: Can the 1,2-migration be controlled? Scope and Limitations. J. Am. Chem. Soc. 2007, 129, 6931-6942.

24. Feuerstein, M.; Doucet, H.; Santelli, M. Efficient Heck vinylation of aryl halides catalyzed by a new air-stable palladium-tetraphosphine complex. J. Org. Chem. 2001, 66, 5923-5925.

25. Yen, S.K.; Koh, L.L., Hahn, F.E., Huynh, H.V.; Hor, T.S.A. Convenient entry to mono- and dinuclear palladium(II) benzothiazolin-2-ylidene complexes and their activities toward Heck coupling. Organometallics 2006, 25, 5105-5112. 
26. Selvakumar, K.; Zapf, A.; Spannenberg, A.; Beller, M. Synthesis of monocarbenepalladium(0) complexes and their catalytic behavior in cross-coupling reactions of aryldiazonium salts. Chem. Eur. J. 2002, 8, 3901-3906.

27. Herrmann, W.A. $N$-heterocyclic carbenes: A new concept in organometallic catalysis. Angew. Chem. Int. Ed. 2002, 41, 1290-1309

28. Glorius, F. N-heterocyclic carbenes in transition metal catalysis. In Top. Organomet. Chem.; Glorius, F., Ed.; Springer-Verlag: Berlin Heidelberg, Germany, 2007; Volume 3, pp. 1-218.

29. Scott, N.M.; Nolan, S.P. Cross-coupling reactions catalyzed by palladium $N$-heterocyclic carbene complexes. In N-Heterocyclic Carbenes in Synthesis; Nolan, S.P., Ed.; Wiley-VCH: Weinheim, Germany, 2006; pp. 55-70.

30. Waltman, A.W.; Grubbs, R.H. A new class of chelating $N$-heterocyclic carbene ligands and their complexes with palladium. Organometallics 2004, 23, 3105-3109.

31. Schutz, J.; Herdtweck, E.; Herrmann, W.A. Synthesis and catalytic application of palladium pyrazolin-3-ylidene complexes. Organometallics 2004, 23, 6084-6086.

32. Kluser, E.; Neels, A.; Albrecht, M. Mild and rational synthesis of palladium complexes comprising C(4)-bound $N$-heterocyclic carbenes. Chem. Commun. 2006, 4495-4497.

33. Karimi B.; Enders, D. New $N$-heterocyclic carbene palladium complex/ionic liquid matrix immobilized on silica: Application as recoverable catalyst for the Heck reaction. Org. Lett., 2006, 8, 1237-1240.

34. Andrus, M.B.; Song C.; Zhang, J. Palladium-imidazolium carbene catalyzed Mizoroki-Heck coupling with aryl diazonium ions. Org. Lett. 2002, 4, 2079-2082.

35. Yang, C.; Lee, H.M.; Nolan, S.P. Highly efficient Heck reactions of aryl bromides with $n$-butyl acrylate mediated by a palladium/phosphine-imidazolium salt system. Org. Lett. 2001, 3, 1511-1514.

36. McGuinness, D.S:, Cavell, K.J.; Skelton, B.W.; White, A.H. Zerovalent palladium and nickel complexes of heterocyclic carbenes: Oxidative addition of organic halides, carbon-carbon coupling processes, and the Heck reaction. Organometallics 1999, 18,1596-1605.

37. Bourissou, D.; Guerret, O.; Gabbai, F.P.; Bertrand, G. Stable carbenes. Chem. Rev. 2000, 100, 39-92.

38. Böhm, V.P.W.; Weskamp, T.; Gstoettmayr, C.W.K.; Herrmann, W.A. Nickel-catalyzed crosscoupling of aryl chlorides with aryl Grignard reagents. Angew. Chem. Int. Ed. Engl. 2000, 39, $1602-1604$.

39. Herrmann, W.A.; Kohlpaintner, C.W. Water-soluble ligands, metal complexes and catalysts: Synergism of homogeneous and heterogeneous catalysis. Angew. Chem. Int. Ed. Engl. 1993, 32, 1524-1544.

40. Schwarz, J.; Herdtweck, E.; Herrmann, W.A.; Gardiner, M.G. Highly efficient monocationic palladacycles of chelating diphosphines in $\mathrm{C}_{2} \mathrm{H}_{4} / \mathrm{CO}$ copolymerization. Organometallics 2000, 19, 3154-3160.

41. Sanford, M.S.; Love, J.A.; Grubbs, R.H. Mechanism and activity of ruthenium olefin metathesis catalysts. J. Am. Chem. Soc. 2001, 123, 6543-6554. 
42. Ahrens, S.; Zeller, A.; Taige, M.A.; Strassner, T. Extension of the alkane bridge in bis NHC-palladium-chloride complexes. Synthesis, structure, and catalytic activity. Organometallics 2006, 25, 5409-5415.

43. Herrmann, W.A:; Öfele, K.; Preysing, D.; Herdtweck, E. Metal complexes of acyclic diaminocarbenes: Links between $\mathrm{N}$-heterocyclic carbene (NHC)- and Fischer-carbene complexes. J. Organomet. Chem. 2003, 684, 235-248.

44. Mayr, M.; Wurst, K.; Ongania, K.H.; Buchmeiser, M.R. 1,3-Dialkyl- and 1,3-diaryl-3,4,5,6tetrahydropyrimidin-2-ylidene rhodium(i) and palladium(II) complexes: Synthesis, structure and reactivity. Chem. Eur. J. 2004, 10, 1256-1266.

45. Özdemir, I.; Demir, S.; Çetinkaya, B. Use of tetrahydropyrimidinium salts for highly efficient palladium-catalyzed cross-coupling reactionsof aryl bromides and chlorides. Tetrahedron 2005, 61, 9791-9798.

46. Schneider, S.K.; Herrmann, W.A.; Herdtweck, E. Active catalysts for the Suzuki coupling: Palladium complexes of tetrahydropyrimid-2-ylidenes. J. Mol. Cat. A 2006, 245, 248-254.

47. Bortenschlager, M.; MAyr, M.; Nuyken, O.; Buchmeiser, M.R. Hydroformylation of 1-octene using rhodium-1,3- $\mathrm{R}_{2}-3,4,5,6$-tetrahydropyrimidin-2-ylidenes $(\mathrm{R}=2$-Pr, mesityl) J. Mol. Cat. 2005, 233, 67-71.

48. Imlinger, N.; Mayr, M.; Wang, D.; Wurst, K.; Buchmeiser, M.R. Arylation of carbonyl compounds catalyzed by rhodium and iridium 1,3-R2-tetrahydropyrimidin-2-ylidenes: Structurereactivity correlations. Adv. Synth. Catal. 2004, 346, 1836-1843.

49. Gürbüz, N.; Özdemir, İ.; Demir, S.; Çetinkaya, B. Improved palladium-catalyzed coupling reaction of aryl halides using saturated $N$-heterocarbene ligands. J. Mol. Cat. A 2004, 209, 23-38.

50. Özdemir, İ.; Demir, S.; Çetinkaya, B. Novel tetrahydropyrimidinium/palladium system as a convenient catalyst: Suzuki coupling reactions of aryl chloride. ARKIVOC 2007, xiii, 71-78.

51. Özdemir, İ.; Gürbüz, N.; Gök, Y.; Çetinkaya, E.; Çetinkaya, B. Palladium-catalyzed SuzukiMiyaura reaction of aryl chlorides in aqueous media using tetrahydrodiazepinium salts as carbene ligands. Synlett 2005, 15, 2394-2396.

52. Özdemir, İ.; Demir, S.; Çetinkaya, B. Palladium-catalyzed Heck reaction of aryl bromides in aqueous media using tris( $N$-heteocyclic carbene) ligands. Synlett 2007, 6, 889-892.

Sample Availability: Samples of the compounds are available from authors .

(C) 2010 by the authors; licensee Molecular Diversity Preservation International, Basel, Switzerland. This article is an open-access article distributed under the terms and conditions of the Creative Commons Attribution license (http://creativecommons.org/licenses/by/3.0/). 